\title{
Lutetium Lu-177 Girentuximab
}

National Cancer Institute

\section{Source}

National Cancer Institute. Lutetium Lu-177 Girentuximab. NCI Thesaurus. Code C66978.

A radioimmunoconjug ate consisting of the chimeric monoclonal antibody cG250 linked to the low energy beta-emitting radioisotope Lutetium 177, via the bifunctional macrocyclic chelating agent tetra-azacyclododecanetetra-acetic acid (DOTA), with potential antineoplastic activity. The antibody moiety of lutetium Lu-177-DOTA-chimeric monoclonal antibody cG250 binds to renal cell carcinoma (RCC) cells expressing the RCCassociated antigen G250; a cytotoxic dose of beta radiation is selectively delivered to G250-expressing RCC cells upon internalization of the radioimmunoconjugate. 